\title{
APPROXIMATION AND THE TOPOLOGY OF RATIONALLY CONVEX SETS
}

\author{
E. S. ZERON
}

\begin{abstract}
Considering a mapping $g$ holomorphic on a neighbourhood of a rational convex set $K \subset \mathbb{C}^{n}$, and range into the complex projective space $\mathbb{C P}^{m}$, the main objective of this paper is to show that we can uniformly approximate $g$ on $K$ by rational mappings defined from $\mathbb{C}^{m}$ into $\mathbb{C P}^{m}$. We only need to ask that the second Čech cohomology group $\check{H}^{2}(K, \mathbb{Z})$ vanishes.
\end{abstract}

\section{INTRODUCTION}

Let $\mathbb{C P}^{m}$ be the $m$-complex projective space, composed of all the complex lines in $\mathbb{C}^{m+1}$ which pass through the origin. It is well known that $\mathbb{C P} \mathbb{P}^{m}$ is a $m$-complex manifold, and that there exists a natural holomorphic projection $\rho_{m}$ defined from $\mathbb{C}^{m+1} \backslash\{0\}$ onto $\mathbb{C P}^{m}$, which sends any point $\left(z_{0}, \ldots, z_{m}\right) \neq$ 0 to the complex line

$$
\rho_{m}\left(z_{0}, \ldots, z_{m}\right)=\left[z_{0}, \ldots, z_{m}\right]:=\left\{\left(z_{0} t, \ldots, z_{m} t\right): t \in \mathbb{C}\right\} .
$$

In particular, we have that the one-dimensional complex projective space $\mathbb{C P}^{1}$ is the Riemann sphere $\mathcal{S}^{2}$, and the natural holomorphic projection $\rho_{1}$ is given by $\rho_{1}(w, z)=\left[\frac{w}{z}, 1\right]$ or $\left[1, \frac{z}{w}\right]$. Thus, any rational mapping $p / q$ defined on $\mathbb{C}^{n}$ may be seen as the composition $\rho_{1}(p, q)$, where $(p, q)$ is a holomorphic polynomial mapping from $\mathbb{C}^{n}$ into $\mathbb{C}^{2}$. The critical set $E$ of $p / q$ is the inverse image $(p, q)^{-1}(0)$, and so $p / q$ is a holomorphic mapping defined from $\mathbb{C}^{n} \backslash E$ into $\mathcal{S}^{2}$. Previous interpretation allows us to extend the notion of rational mapping to consider the natural projections $\rho_{m}$ for $m \geq 1$.

Definition 1. A rational mapping based on $\mathbb{C}^{n}$, and image in $\mathbb{C P}^{m}$, is defined as the composition $\rho_{m}(P)$, for a given holomorphic polynomial mapping $P: \mathbb{C}^{n} \rightarrow \mathbb{C}^{m+1}$. The critical set $E$ of $\rho_{m}(P)$ is then defined as the inverse image $P^{-1}(0)$, and so $\rho_{m}(P)$ is a holomorphic mapping defined from $\mathbb{C}^{n} \backslash E$ into $\mathbb{C P}^{m}$.

On the other hand, recalling the fundamentals of rational approximation theory. We have that a compact set $K$ in $\mathbb{C}^{n}$ is rationally convex if for every point $y \in \mathbb{C}^{n} \backslash K$ there exists a holomorphic polynomial $p$ such that

Date: March 11, 2021.

1991 Mathematics Subject Classification. 32E30 or 32Q55.

Key words and phrases. Rationally convex, cohomology and homotopy.

Research supported by Cinvestav and Conacyt, México. 
$p(y)=0$ and $p$ does not vanishes on $K$. Besides, it is well known that each function $h: U \rightarrow \mathbb{C}$ holomorphic on a neighbourhood $U$ of $K$ can be approximated on $K$ by rational functions, whenever $K$ is rationally convex, see for example 2] or [7]. That is, for each $\widehat{\delta}>0$ there exists a holomorphic rational function $p / q$ such that $K$ does not meet the zero locus of $q$ and $\left|\frac{p(z)}{q(z)}-h(z)\right|$ is strictly less that $\widehat{\delta}$ on $K$. This result automatically drives us to consider whether the concept of rationally convex sets is strong enough as to imply approximation by the kind of rational mappings that we have just introduced on Definition 1. Amazingly, we can get a positive answer by adding a simple cohomological condition.

Theorem 2. (main theorem) Let $K$ be a rationally convex set in $\mathbb{C}^{n}$, and Dist be a metric on $\mathbb{C P}^{m}$ which induces the topology, with $m, n \geq 1$. If the second $\check{C}$ ech cohomology group $\check{H}^{2}(K, \mathbb{Z})$ vanishes. Then, for each $\widehat{\delta}>0$ and any mapping $g: U \rightarrow \mathbb{C P}^{m}$ holomorphic on a neighbourhood $U$ of $K$, there exists a rational mapping $\rho_{m}(P)$ defined on $\mathbb{C}^{m}$, whose critical set does not meet $K$, and such that $\operatorname{Dist}\left[\rho_{m}(P(z)), g(z)\right]$ is less than $\widehat{\delta}$ on $K$.

This result was mainly inspired on the work done by professors Grauert, Kerner and Oka in [9, 10] and [17. Besides, in an early paper [8] we have previously analysed the approximation by the rational mappings described in Definition 1 we deduced a result similar to main theorem by using the extra topological condition of being null-homotopic. We are giving a complete reference to this early result in section 4 .

We shall prove the main theorem in the third section of this paper. Moreover, we are going to devote the second section to introduce the results on cohomology theory that we need for the proof of the main theorem. Finally, examples and corollaries are introduced on section 4 .

\section{Cohomology}

We strongly recommend the bibliography [1], 3] and [1], for references on homotopy theory; and the bibliography [14, [18] and [19], for references on cohomology theory.

We consider two main classes of cohomology groups: Čech and singular. And these cohomology groups are both isomorphic on smooth manifolds, and open subsets of $\mathbb{C}^{n}$, for these spaces are all locally contractible, see for example [18, pp. 166] or [19, pp. 334 and 341]. However, there is a very nice example in [19, p. 77 and 317] of a compact set $K \subset \mathbb{C}$ whose Čech cohomology group $\check{H}^{1}(K, \mathbb{Z})=\mathbb{Z}$, but its singular cohomology group $H_{s}^{1}(K, \mathbb{Z})$ vanishes. Now then, given a closed set $E \subset \mathbb{C}^{n}$, we need Cech cohomology groups because $\check{H}^{*}(E, \mathbb{Z})$ can be calculated as the direct limit of the sequence $\left\{\check{H}^{*}(U, \mathbb{Z})\right\}$, where $U$ runs over a system (directed by inclusions) of open neighbourhoods of $E$ in $\mathbb{C}^{n}$, see for example [2, chapter 15], 3, p. 348], [18, p. 145] or [19, p. 327]. Whence, the Cech cohomology group $\check{H}^{*}(E, \mathbb{Z})$ vanishes if and only if for each element $\xi \in \check{H}^{*}(U, \mathbb{Z})$ defined on 
an open neighbourhood $U$ of $E$, there exists a second open set $W$ such that $E \subset W \subset U$ and the restriction $\left.\xi\right|_{W}$ is equal to zero in $\check{H}^{*}(W, \mathbb{Z})$.

On the other hand, we need singular cohomology groups because of the following universal result. Let $U \subset \mathbb{C}^{n}$ be an open subset which has the homotopy type of a $\mathrm{CW}$-complex, the singular cohomology group $H_{s}^{k}(U, \mathbb{Z})$ is then isomorphic to the group of homotopy classes $[U, Y]$, where $k \geq 1$ and $Y$ is an Eilenberg-McLane space of type $(\mathbb{Z}, k)$, see for example [1, p. 183], 3. pp. 488-492], 4, p. 274] or [19, p. 428]. Recall that $Y$ is an EilenbergMcLane space of type $(\mathbb{Z}, k)$ if every homotopy group $\pi_{*}(Y)$ vanishes, but $\pi_{k}(Y)$ which is equal to $\mathbb{Z}$. Besides, recall that $[U, Y]$ is the group composed by all the homotopy classes of continuous mappings $f: U \rightarrow Y$.

Combining the ideas presented in previous paragraphs, we may deduce the following result. Let $E \subset \mathbb{C}^{n}$ be a closed set whose Cech cohomology group $\check{H}^{k}(E, \mathbb{Z})$ vanishes, $k \geq 1$. Besides, suppose from now on that $E$ has a system (directed by inclusions) of open neighbourhoods $\left\{U_{\beta}\right\}$ in $\mathbb{C}^{n}$, where each $U_{\beta}$ has the homotopy type of a CW-complex and $E=\bigcap_{\beta} U_{\beta}$. Given an Eilenberg-McLane space $Y$ of type $(\mathbb{Z}, k)$, and since Čech and singular cohomology groups are isomorphic on each $U_{\beta}$, we have that for every continuous mapping $f: U_{\beta} \rightarrow Y$ there exists a second neighbourhood $U_{\theta}$ such that $E \subset U_{\theta} \subset U_{\beta}$ and the restriction $\left.f\right|_{U_{\theta}}: U_{\theta} \rightarrow Y$ is nullhomotopic. The main idea behind this result is to see the homotopy class of the mapping $f$ as an element of $H_{s}^{k}\left(U_{\beta}, \mathbb{Z}\right) \cong \check{H}^{k}\left(U_{\beta}, \mathbb{Z}\right)$.

Let us illustrate previous result with a known example. Recalling that $\mathbb{C} \backslash\{0\}$ has the homotopy type of the 1-dimensional sphere $\mathcal{S}^{1}$, and that $\mathcal{S}^{1}$ is a Eilenberg-McLane space of type $(\mathbb{Z}, 1)$, because $\pi_{1}\left(\mathcal{S}^{1}\right)=\mathbb{Z}$ and $\pi_{k}\left(\mathcal{S}^{1}\right)=0$ for every $k \neq 1$. We may deduce that for each non-vanishing continuous function $f: U_{\beta} \rightarrow \mathbb{C} \backslash\{0\}$, and whenever $\check{H}^{1}(E, \mathbb{Z})=0$, there exists a second neighbourhood $U_{\theta}$ such that the restriction $\left.f\right|_{U_{\theta}}: U_{\theta} \rightarrow \mathbb{C} \backslash\{0\}$ has a well defined continuous logarithm $\left(\left.f\right|_{U_{\theta}}\right.$ is null-homotopic). This result was presented in chapter 15 of [2] and in section 7, chapter III, of [7].

Coming back to the main theorem of this paper, we are supposing in the hypotheses that the second $\check{C}$ ech cohomology group $\check{H}^{2}(K, \mathbb{Z})$ vanishes, so we need an example of an Eilenberg-McLane space of type $(\mathbb{Z}, 2)$. This example is given by the infinite dimensional complex projective space $\mathbb{C P}^{\infty}$, see for example [1, p. 360], [11, p. 157] or [19, p. 425]. Making again all the calculations done in previous paragraphs, we may prove the following lemma.

Lemma 3. Let $E$ be a closed subset of $\mathbb{C}^{n}$ whose second Čech cohomology group $\check{H}^{2}(E, \mathbb{Z})$ vanishes. Supposing that $E$ has a system (directed by inclusions) of open neighbourhoods $\left\{U_{\beta}\right\}$ in $\mathbb{C}^{n}$, where each $U_{\beta}$ has the homotopy type of a $C W$-complex and $E=\bigcap_{\beta} U_{\beta}$. We have that for every continuous mapping $f: U_{\beta} \rightarrow \mathbb{C P}^{\infty}$ there exists a second neighbourhood $U_{\theta}$ such that $E \subset U_{\theta} \subset U_{\beta}$ and the restriction $\left.f\right|_{U_{\theta}}: U_{\theta} \rightarrow \mathbb{C P}^{\infty}$ is null-homotopic.

We may now prove the main theorem of this paper. 


\section{Proof of MAIN THEOREM}

We need to recall some properties about the infinite dimensional complex projective space $\mathbb{C P}^{\infty}$. Consider the infinite dimensional space $\mathbb{C}^{\infty}$ composed of all the complex sequences $\left(z_{0}, z_{1}, \ldots\right)$, where only a finite number of entries $z_{k}$ is different from zero. This space $\mathbb{C}^{\infty}$ is naturally endowed with the standard norm $\sqrt{\sum_{k}}\left|z_{k}^{2}\right|$. The complex projective space $\mathbb{C P}^{\infty}$ is then composed of all the complex lines in $\mathbb{C}^{\infty}$ which pass through the origin. Besides, there exists a natural projection $\rho_{\infty}$ defined from $\mathbb{C}^{\infty} \backslash\{0\}$ onto $\mathbb{C P}^{\infty}$ which send any point $\left(z_{0}, z_{1}, \ldots\right) \neq 0$ to the complex line

$$
\rho_{\infty}\left(z_{0}, z_{1}, \ldots\right)=\left[z_{0}, z_{1}, \ldots\right]:=\left\{\left(z_{0} t, z_{1} t, \ldots\right): t \in \mathbb{C}\right\} .
$$

Finally, it is easy to calculate that $\rho_{\infty}$ induces a locally trivial fibre bundle in $\mathbb{C}^{\infty} \backslash\{0\}$, with base on $\mathbb{C P}^{\infty}$ and fibre $\mathbb{C} \backslash\{0\}$, see [1, p. 360]. We may cover $\mathbb{C P}^{\infty}$ with open sets $W_{k}$ composed of all points in $\mathbb{C P}^{\infty}$ whose $k$ entry is equal to one. The open set $W_{0}$ is equal to $\left\{[1, y]: y \in \mathbb{C}^{\infty}\right\}$, for example. It is now easy to calculate that $\rho_{\infty}$ induces a trivial fibre bundle on each $\rho_{\infty}^{-1}\left(W_{k}\right)$, with base on $W_{k}$ and fibre $\mathbb{C} \backslash\{0\}$. Actually, for every $m \geq 1$, we have that the projection $\rho_{m}$ induces a locally trivial fibre bundle in $\mathbb{C}^{m+1} \backslash\{0\}$, with base on $\mathbb{C P}^{m}$ and fibre $\mathbb{C} \backslash\{0\}$, as well. We may now prove the main theorem.

Proof. (main theorem). Define the open rational polyhedra $V_{\beta}$ in $\mathbb{C}^{n}$ by the formula,

$$
V_{\beta}:=\left\{z \in \mathbb{C}^{n}:\left|p_{j}(z)\right|<1, \forall j, \text { and }\left|q_{k}(z)\right|>1, \forall k\right\},
$$

for some given collections of holomorphic polynomials $\left\{p_{j}\right\}$ and $\left\{q_{k}\right\}$ in $\mathbb{C}^{n}$. We have that each rational polyhedron $V_{\beta}$ is an open Stein subset of $\mathbb{C}^{n}$, so $V_{\beta}$ has the homotopy type of a CW-complex [15, p. 39]. Moreover, given a rationally convex set $K \subset \mathbb{C}^{n}$, it is easy to see that the family of all open rational polyhedra $V_{\beta}$ which contain to $K$ form a system of neighbourhoods in $\mathbb{C}^{n}$, and that $K$ is equal to the intersection $\bigcap_{K \subset V_{\beta}} V_{\beta}$.

On the other hand, let $g: U \rightarrow \mathbb{C P}^{m}$ be any mapping holomorphic on a neighbourhood $U$ of $K$. We can obviously extend this mapping to a second one with range on $\mathbb{C P}^{\infty}$, we only need to set the first $m+1$ entries equal to the entries of $g=\left[g_{0}, \ldots, g_{m}\right]$ and the rest of them equal to zero. That is, define $\breve{g}: U \rightarrow \mathbb{C P} \mathbb{P}^{\infty}$ by

$$
\breve{g}(z):=\left[g_{0}(z), \ldots, g_{m}(z), 0, \ldots, 0, \ldots\right] .
$$

We may find an open rational polyhedron $V_{\beta}$ such that $K \subset V_{\beta} \subset U$. Besides, recalling that the Čech cohomology group $\check{H}^{2}(K, \mathbb{Z})$ vanishes because the given hypotheses, and considering Lemma 3, we may even find a rational polyhedron $V_{\beta}$ such that the restriction $\left.\breve{g}\right|_{V_{\beta}}: V_{\beta} \rightarrow \mathbb{C P}^{\infty}$ is null-homotopic. Let $I=[0,1]$ the unit closed interval in the real line. There exists then a continuous mapping $G$ from $V_{\beta} \times I$ into $\mathbb{C P}^{\infty}$ such that $G(z, 1)=\breve{g}(z)$ and 
$G(z, 0)=[1,0, \ldots]$, for every $z \in V_{\beta}$. We have the following commutative diagram,

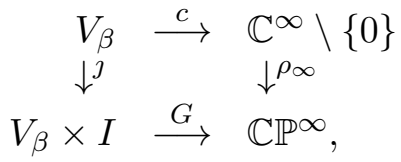

where $c(z)=(1,0, \ldots)$ is a constant mapping and $\jmath(z)=(z, 0)$ is the natural inclusion. We know that the projection $\rho_{\infty}$ induces a locally trivial fibre bundle on $\mathbb{C}^{\infty} \backslash\{0\}$, with base $\mathbb{C P}^{\infty}$ and fibre $\mathbb{C} \backslash\{0\}$. This fibre bundle has the homotopy lifting property, see for example [4, pp. 62 and 67], [11, p. 87] or [19, p. 96]. Hence, there exists a continuous mapping $F$ from $V_{\beta} \times I$ into $\mathbb{C}^{\infty} \backslash\{0\}$ such that $\rho_{\infty}(F)$ is identically equal to $G$ on $V_{\beta} \times I$. Recalling equation (2), where $\breve{g}(z)=G(z, 1)$ was defined, we can deduce that $F(z, 1)$ has the form,

$$
F(z, 1):=\left(F_{0}(z, 1), \ldots, F_{m}(z, 1), 0, \ldots, 0, \ldots\right) .
$$

We may then introduce a new continuous mapping $f$ defined from $V_{\beta}$ into $\mathbb{C}^{m+1} \backslash\{0\}$, by removing the last entries of $F(z, 1)$ equal to zero; that is,

$$
f(z):=\left(F_{0}(z, 1), \ldots, F_{m}(z, 1)\right) \text { for every } z \in V_{\beta} .
$$

It is easy to see that $\rho_{m}(f(z))=g(z)$ for every $z \in V_{\beta}$. The main objective of previous calculations was the construction of the continuous mapping $f$ described above. Actually, we could have showed the existence of such a mapping $f$ by using the results on obstruction theory described in [3. p. 507] and [19, p. 447]. However, we think that the procedure followed in previous paragraphs is much more simple and illustrative.

Nevertheless, we look for a holomorphic (not only continuous) mapping $h$ from $V_{\beta}$ into $\mathbb{C}^{m+1} \backslash\{0\}$ such that $\rho_{m}(h(z))=g(z)$ for every $z \in V_{\beta}$. We shall construct this holomorphic mapping $h$ by using Oka's results on the second Cousin problem. Define the space,

$$
M:=\left\{(z, w) \in V_{\beta} \times \mathbb{C}^{m+1}: g(z)=\rho_{m}(w), w \neq 0\right\} .
$$

It is easy to deduce that $M$ is an analytic space because $g$ and $\rho_{m}$ are both holomorphic mappings. Moreover, we also have the following commutative diagram,

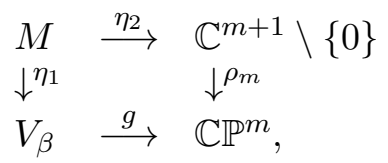

where $\eta_{1}(z, w)=z$ and $\eta_{2}(z, w)=w$ are the basic projections. It is easy to prove that $\eta_{1}$ induces a locally trivial fibre bundle in $M$, with Stein base on $V_{\beta}$ and Stein fibre $\mathbb{C} \backslash\{0\}$. This fibre bundle $M \stackrel{\eta_{1}}{\longrightarrow} V_{\beta}$ is the pull back of the fibre bundle induced by $\rho_{m}$ on $\mathbb{C}^{m+1} \backslash\{0\}$. Now then, recalling the continuous mapping $f$ defined above, we automatically have that $z \mapsto(z, f(z))$ is a continuous section of the fibre bundle $M \stackrel{\eta_{1}}{\longrightarrow} V_{\beta}$, because $g(z)=\rho_{m}(f(z))$ for every $z \in V_{\beta}$. Oka's results on the second 
Cousin problem imply that $z \mapsto(z, f(z))$ is homotopic to a holomorphic section $z \rightarrow(z, h(z))$, because $V_{\beta}$ is Stein, see for example [5], 6], 10] or [17. Hence, there exists a holomorphic mapping $h: V_{\beta} \rightarrow \mathbb{C}^{m+1} \backslash\{0\}$ such that $g(z)$ is equal to $\rho_{m}(h(z))$ for every $z \in V_{\beta}$.

On the other hand, Let $W$ be an open subset of $\mathbb{C}^{m+1}$ which contains the compact image $h(K)$. Besides, suppose that the closure $\bar{W}$ is compact and does not contains the origin. Notice that the projection $\rho_{m}$ from $\mathbb{C}^{m+1} \backslash\{0\}$ into $\mathbb{C P}^{m}$ is continuous with respect to the metric Dist, which induces the topology, so $\rho_{m}$ is also uniformly continuous on $\bar{W}$. Express $h=\left(h_{0}, \ldots, h_{m}\right)$ as a vector in $\mathbb{C}^{m+1}$. There are $m+1$ small enough constants $\delta_{k}>0$ such that, given $z \in K$ and $w \in \mathbb{C}^{m+1}$,

$$
\begin{gathered}
\operatorname{Dist}\left[\rho_{m}(h(z)), \rho_{m}(w)\right]<\widehat{\delta} \quad \text { and } \quad w \in \bar{W}, \\
\text { whenever }\left|h_{k}(z)-w_{k}\right|<\delta_{k} \quad \text { for } \quad 0 \leq k \leq m .
\end{gathered}
$$

Recalling that $K$ is rationally convex, we may find $m+1$ rational functions $w_{k}=\frac{p_{k}}{q_{k}}$ defined on $\mathbb{C}^{n}$, such that $K$ meets the zero locus of no $q_{k}$, for every $0 \leq k \leq m$, and the absolute value $\left|h_{k}(z)-\frac{p_{k}}{q_{k}}(z)\right|$ is strictly less than $\delta_{k}$ on $K$. Consider the polynomial mapping $P: \mathbb{C}^{n} \rightarrow \mathbb{C}^{m+1}$ given by

$$
P:=\left(\frac{p_{0}}{q_{0}} \prod_{k=0}^{m} q_{k}, \ldots, \frac{p_{m}}{q_{m}} \prod_{k=0}^{m} q_{k}\right) .
$$

It is easy to deduce that neither $\left(\frac{p_{0}}{q_{0}}, \ldots, \frac{p_{m}}{q_{m}}\right)$ nor the product $\prod_{k=0}^{m} q_{k}$ can vanishes on $K$, because $0 \notin \bar{W}$ and equation 3 . Thus, the compact set $K$ does not meet the critical set $P^{-1}(0)$ of the rational mapping $\rho_{m}(P)$. We may also deduce that $\rho_{m}(P)$ is equal to $\rho_{m}\left(\frac{p_{0}}{q_{0}}, \ldots, \frac{p_{m}}{q_{m}}\right)$ on $K$. Therefore, recalling equation 3 and that $\rho_{m}(h(z))$ is equal to $g(z)$ for every $z \in K$, we get the result that we look for: the distance Dist $\left[g(z), \rho_{m}(P(z))\right]$ is strictly less that $\widehat{\delta}$ on $K$.

\section{EXAMPLES AND APPLICATIONS}

We shall conclude this paper by noticing that the cohomological condition $\breve{H}^{2}(K, \mathbb{Z})=0$ in the hypotheses of the main theorem is not a trivial condition. Consider the standard two-sphere in $\mathbb{R}^{3}$,

$$
\mathcal{S}^{2}:=\left\{(a, b, c) \in \mathbb{R}^{3}: a^{2}+b^{2}+c^{2}=1\right\} .
$$

We can obviously analyse $\mathcal{S}^{2}$ as a subset of $\mathbb{C}^{3}$, embedding it into the real axis; and it is easy to see that $\mathcal{S}^{2}$ is rationally convex in $\mathbb{C}^{3}$. Besides, the groups $\check{H}^{2}\left(\mathcal{S}^{2}, \mathbb{Z}\right)$ and $H_{s}^{2}\left(\mathcal{S}^{2}, \mathbb{Z}\right)$ are both isomorphic to $\mathbb{Z}$. Finally, consider the following open neighbourhood $U$ of $\mathcal{S}^{2}$,

$$
U:=\left\{(x, y, z) \in \mathbb{C}^{3}:-\pi<\arg \left(x^{2}+y^{2}+z^{2}\right)<\pi\right\} .
$$

And the holomorphic mapping $g: U \rightarrow \mathbb{C P}^{1}$, where $\sqrt{1}=1$,

$$
g(x, y, z)=\left[\frac{x+i y}{\sqrt{ }\left(x^{2}+y^{2}+z^{2}\right)+z}, 1\right] \quad \text { or } \quad\left[1, \frac{x-i y}{\sqrt{ }\left(x^{2}+y^{2}+z^{2}\right)-z}\right] .
$$


It is easy to see that the restriction $\left.g\right|_{\mathcal{S}^{2}}$ is the identity mapping from $\mathcal{S}^{2}$ onto $\mathbb{C P}^{1}$, because $g(a, b, c)=\left[\frac{a+i b}{1+c}, 1\right]$ is the stereographic projection, so $\left.g\right|_{\mathcal{S}^{2}}$ is not null-homotopic. Moreover, we assert that $g$ cannot be approximated on $\mathcal{S}^{2}$ by rational mappings $\rho_{1}(P)$. That is, there exists a fix constant $\beta_{g}>0$, such that for every rational mapping $\rho_{1}(P)$ holomorphic on $\mathcal{S}^{2}$ there is a point $\breve{w} \in \mathcal{S}^{2}$ with $\operatorname{Dist}\left[\rho_{1}(P(\breve{w})), g(\breve{w})\right]$ greater than $\beta_{g}$.

Let $P: \mathbb{C}^{3} \rightarrow \mathbb{C}^{2}$ be any polynomial mapping whose fibre $P^{-1}(0)$ does not meet $\mathcal{S}^{2}$. We can deduce that the restriction $\left.P\right|_{\mathcal{S}^{2}}$ defined from $\mathcal{S}^{2}$ into $\mathbb{C}^{2} \backslash\{0\}$ is null-homotopic, because $\mathbb{C}^{2} \backslash\{0\}$ has the homotopy type of the three-sphere $\mathcal{S}^{3}$ and the second homotopy group $\pi_{2}\left(\mathcal{S}^{3}\right)$ vanishes. Whence, we also have that the restriction $\left.\rho_{1}(P)\right|_{\mathcal{S}^{2}}$ is null-homotopic for every rational mapping $\rho_{1}(P)$ defined on $\mathbb{C}^{3}$, and whose critical set $P^{-1}(0)$ does not meet $\mathcal{S}^{2}$. Finally, since $\left.g\right|_{\mathcal{S}^{2}}$ is not null-homotopic, we can conclude that there exists a fix constant $\beta_{g}>0$, such that for every rational mapping $\rho_{1}(P)$ holomorphic on $\mathcal{S}^{2}$ there is a point $\breve{w} \in \mathcal{S}^{2}$ with $\operatorname{Dist}\left[\rho_{1}(P(\breve{w})), g(\breve{w})\right]$ greater than $\beta_{g}$. We only need to recall that $\mathbb{C P}^{1}$ is an absolute neighbourhood retract [13, pp. 332 and 339], and to apply the following lemma which was originally presented in 8 .

Lemma 4. Let $X$ and $(Y, \mathrm{~d})$ be two metric spaces, such that $X$ is compact and $Y$ is an absolute neighbourhood retract. Then, given a fixed continuous mapping $g: X \rightarrow Y$, there exists a constant $\beta_{g}>0$ such that: every continuous mapping $f: X \rightarrow Y$ is homotopic to $g$, whenever $\mathrm{d}[g(x), f(x)]$ is less than $\beta_{g}$ for every $x \in X$.

Proof. Let $Y^{X}$ be the topological space composed of all the continuous mappings $f: X \rightarrow Y$, and endowed with the compact-open topology. Since $X$ is compact and $(Y, \mathrm{~d})$ is metric, the compact-open topology of $Y^{X}$ is induced by the metric

$$
\mathrm{D}\left[f_{1}, f_{2}\right]:=\sup \left\{\mathrm{d}\left[f_{1}(x), f_{2}(x)\right]: x \in X\right\},
$$

for any two mappings $f_{1}$ and $f_{2}$ in $Y^{X}$ (see for example [13, p. 89]). The space $Y^{X}$ is locally arcwise connected and an absolute neighbourhood retract, because $Y$ is an absolute neighbourhood retract (see [13, pp. 339340]). Whence, there exists a fixed constant $\beta_{g}>0$ such that the open ball in $Y^{X}$ with centre in $g$ and radius $\beta_{g}$ is contained in an arcwise connected neighbourhood of $g$. That is, for every continuous mapping $f: X \rightarrow Y$ with $\mathrm{D}[f, g]<\beta_{g}$, there exists an arc in $Y^{X}$ whose end points are $f$ and $g$; and so the mappings $g$ and $f$ are homotopic.

Finally, the ideas introduced in the proof of the main theorem may be used to show several versions of this theorem. For example, given a closed set $E$ in $\mathbb{C}^{n}$, a continuous function $f: E \rightarrow \mathbb{C}$ can be tangentially approximated by meromorphic ones if for every strictly positive continuous function $\hat{\epsilon}: E \rightarrow \mathbb{R}$ there exists a pair of holomorphic functions $\phi$ and $\psi$ defined from $\mathbb{C}^{n}$ into 
$\mathbb{C}$, such that $E$ does not meet the zero locus of $\psi$ and $\left|f(z)-\frac{\phi(z)}{\psi(z)}\right|$ is less than $\hat{\epsilon}(z)$ for every $z \in E$. We may show the following result now.

Theorem 5. Let $E$ be a closed set in $\mathbb{C}^{n}$ whose second Cech cohomology group $\check{H}^{2}(E, \mathbb{Z})$ vanishes, and such that every continuous function $f: E \rightarrow$ $\mathbb{C}$ can be tangentially approximated by meromorphic functions. Suppose that Dist is a metric on $\mathbb{C P}^{m}$ which induces the topology, and that $E$ has a system of open neighbourhoods $\left\{V_{\beta}\right\}$ in $\mathbb{C}^{n}$, where each $V_{\beta}$ has the homotopy type of a $C W$-complex and $E=\bigcap_{\beta} V_{\beta}$.

For every pair of continuous mappings $\xi: E \rightarrow \mathbb{C P}^{m}$ and $\hat{\epsilon}: E \rightarrow \mathbb{R}$, with $\hat{\epsilon}(z)>0$, there exists a holomorphic mapping $H$ defined from $\mathbb{C}^{n}$ into $\mathbb{C}^{m+1}$, such that $E$ does not meet the zero locus of $H$ and $\operatorname{Dist}\left[\rho_{m}(H(z)), \xi(z)\right]$ is less than $\hat{\epsilon}(z)$ for every $z \in E$.

Proof. We only give a sketch of this proof, for it is essentially the same one presented in section three. Firstly, we have that $\mathbb{C P}^{m}$ is an absolute neighbourhood retract, for it is homeomorphic to a compact polyhedron, see [13, pp. 332 and 339]. Therefore, there exists a continuous mapping $g: U \rightarrow \mathbb{C P}^{m}$ defined on an open neighbourhood $U$ of $E$ and such that $g(z)=\xi(z)$ for every $z \in E$. Besides, following equation (2), we can extend $g$ to a continuous mapping $\breve{g}$ defined from $U$ into $\mathbb{C P}^{\infty}$.

Considering Lemma 3, there exists an open neighbourhood $V_{\beta}$ such that $E \subset V_{\beta} \subset U$ and the restriction $\left.\breve{g}\right|_{V_{\beta}}: V_{\beta} \rightarrow \mathbb{C P}^{\infty}$ is null-homotopic. Following the ideas presented in pages 4 and 5 of this paper, we can build a second continuous (not necessarily holomorphic) mapping $h$ from $V_{\beta}$ into $\mathbb{C}^{m+1} \backslash\{0\}$ such that $\rho_{m}(h(z))$ is equal to $g(z)=\xi(z)$ for every $z \in E$. Notice that $\rho_{m}$ is continuous with respect to the metric Dist, which induces the topology. Express $h=\left(h_{0}, \ldots, h_{m}\right)$ as a vector in $\mathbb{C}^{m+1} \backslash\{0\}$. There are $m+1$ strictly positive continuous functions $\delta_{k}: E \rightarrow \mathbb{R}$ such that, given $z \in E$ and $w \in \mathbb{C}^{m+1}$,

$$
\begin{array}{r}
\text { Dist }\left[\rho_{m}(h(z)), \rho_{m}(w)\right]<\hat{\epsilon}(z) \quad \text { whenever } \\
\left|h_{k}(z)-w_{k}\right|<\delta_{k}(z) \text { for } \quad 0 \leq k \leq m .
\end{array}
$$

Recalling that every continuous function can be tangentially approximated by meromorphic functions on $E$, we may find holomorphic functions $\phi_{k}$ and $\psi_{k}$ defined from $\mathbb{C}^{n}$ into $\mathbb{C}$ such that $E$ meets the zero locus of no $\psi_{k}$, for $0 \leq k \leq m$, and the absolute value $\left|h_{k}(z)-\frac{\phi_{k}}{\psi_{k}}(z)\right|$ is strictly less than $\delta_{k}(z)$ for every $z \in E$. Moreover, we can even choose the functions $\delta_{k}$ in such a way that each $\delta_{k}(z)$ is strictly less than $\max \left\{\frac{\left|h_{j}(z)\right|}{2}\right\}$ for all $z \in E$. Hence, we have that neither $\left(\frac{\phi_{0}}{\psi_{0}}, \ldots, \frac{\phi_{m}}{\psi_{m}}\right)$ nor the product $\prod_{k=0}^{m} \psi_{k}$ can vanishes on $E$; and so, the set $E$ does not meet the zero locus of the holomorphic mapping $H: \mathbb{C}^{n} \rightarrow \mathbb{C}^{m+1}$ defined by,

$$
H:=\left(\frac{\phi_{0}}{\psi_{0}} \prod_{k=0}^{m} \psi_{k}, \ldots, \frac{\phi_{m}}{\psi_{m}} \prod_{k=0}^{m} \psi_{k}\right) .
$$


It is easy to deduce that $\rho_{m}(H)$ is equal to $\rho_{m}\left(\frac{\phi_{0}}{\psi_{0}}, \ldots, \frac{\phi_{m}}{\psi_{m}}\right)$ on $E$. Whence, recalling equation 4 , and that $\rho_{m}(h(z))$ is equal to $g(z)=\xi(z)$ for every $z \in E$, we get the result that we look for: $\operatorname{Dist}\left[\xi(z), \rho_{m}(H(z))\right]$ is strictly less than $\hat{\epsilon}(z)$ on $E$.

We have already proved a weaker version of this theorem in 8 . We used there the stronger hypotheses that $E$ is compact and $\xi: E \rightarrow \mathbb{C P}^{m}$ is null-homotopic, instead of asking $\check{H}^{2}(E, \mathbb{Z})=0$.

\section{REFERENCES}

1. M.A. Aguilar, S. Gitler and C. Prieto. Topología algebraica, un enfoque homotópico. McGraw-Hill, México, 1998.

2. H. Alexander and J. Wermer. Several Complex Variables and Banach Algebras, third edition, (Graduate Texts in Mathematics, 35). Springer-Verlag, New York, 1998.

3. Glen E. Bredon. Topology and geometry, (Graduate Texts in Mathematics, 139). Springer-Verlag, New York, 1993.

4. C.T.J. Dodson and P.E. Parker. A user's guide to algebraic topology. Kluwer Academic Publishers, Dordrecht the Netherlands, 1997.

5. F. Forstnerič. Oka's principle for sections of subelliptic submersions. Math. Z. 241 (2002), pp. 527-551.

6. F. Forstnerič and J. Prezelj. Oka's principle for holomorphic submersions with sprays. Math. Ann. 322 (2002), pp. 633-666.

7. T.W. Gamelin. Uniform algebras. Prentice-Hall, Englewood Cliffs N.J., 1969.

8. Paul M. Gauthier and E. S. Zeron. Approximation by Rational Mappings, via Homotopy Theory. Canad. Math. Bull. in press.

9. H. Grauert. Holomorphe Funktionen mit Werten in komplexen Lieschen Gruppen. Math. Ann. 133 (1957), pp. 450-472.

10. H. Grauert and H. Kerner. Approximation von holomorphen Schnittflächen in Faserbündeln mit homogener Faser. Arch. Math. 14 (1963), pp. 328-333.

11. B. Gray. Homotopy theory, an introduction to algebraic topology. Academic Press, New York, 1975.

12. L. Hörmander and J. Wermer. Uniform approximation on compact sets in $\mathbb{C}^{n}$. Math. Scand. 23 (1968), pp. 5-21.

13. K. Kuratowski. Topology, Vol. II. Academic Press, New York and London, 1968.

14. W.S. Massey. Homology and cohomology theory. Marcel Dekker, New York, 1978.

15. J. Milnor. Morse theory, (Annals of Mathematics Studies, 51). Princeton University Press, Princeton N.J., 1963.

16. R. Nirenberg and R. O. Wells Jr.. Approximation theorems on differentiable submanifolds of a complex manifold. Trans. Amer. Math. Soc. 142 (1969) pp. 15-35.

17. K. Oka. Sur les fonctions des plusieurs variables III: Deuxième problème de Cousin. J. Sc. Hiroshima Univ. 9 (1939), pp. 7-19.

18. E. G. Sklyarenko. Homology and cohomology theories of general spaces. (General topology, II, Encyclopaedia Math. Sci., 50), Springer, Berlin, 1996, pp. 119-256.

19. E. H. Spanier. Algebraic topology. McGraw-Hill, New York-Toronto, 1966.

Depto. Matemáticas, CivestaV, Apdo. Postal 14-740, México DF, 07000, MÉXICO.

E-mail address: eszeron@math.cinvestav.mx

Centre de Recherches Mathématiques, Université de Montréal, Case postale 6128, Succursale centre-ville, Montréal, H3C 3J7, Canada. 